\title{
Homogeneous and isotropic big rips?
}

\author{
Massimo Giovannini* \\ Centro "Enrico Fermi”, Compendio del Viminale, Via Panisperna 89/A, 00184 Rome, Italy \\ Department of Physics, Theory Division, CERN, 1211 Geneva 23, Switzerland
}

(Received 15 July 2005; published 14 October 2005)

\begin{abstract}
We investigate the way big rips are approached in a fully inhomogeneous description of the space-time geometry. If the pressure and energy densities are connected by a (supernegative) barotropic index, the spatial gradients and the anisotropic expansion decay as the big rip is approached. This behavior is contrasted with the usual big-bang singularities. A similar analysis is performed in the case of sudden (quiescent) singularities and it is argued that the spatial gradients may well be non-negligible in the vicinity of pressure singularities.
\end{abstract}

DOI: 10.1103/PhysRevD.72.083508

PACS numbers: 98.80.Cq, 98.80.- $\mathrm{k}$

Consider the situation where the matter content of the present Universe is dominated by a perfect fluid with barotropic index $w=p / \rho<-1$. Evidence of this possibility seems to be suggested from the analysis of Type Ia supernovae. If this is the case, future singularities may be expected [1,2] (see also Refs. [3-5]).

In the present paper we intend to study the nature of future big-rip singularities in a fully inhomogeneous approach whose relevance in the context of usual big-bang singularities had been exploited long ago [6] (see also [7,8] and references therein). For instance, in the case of conventional big-bang singularities, one can show that the relative contribution of the gradients decays as the singularity is approached. Does the same happen in the case of future (big-rip) singularities? In the case of big-bang singularity, the anisotropy is believed to play an important role in the way the singularity is effectively approached. Is this true also for big-rips? These are some of the questions we ought to address.

Consider first the case where the perfect barotropic fluid filling the Universe is characterized by a supernegative equation of state, i.e. $w=-1-\epsilon$, with $\epsilon>0$. We shall then be interested in the contribution of the spatial gradients as the big rip is approached. To achieve this goal Einstein equations must be written in fully inhomogeneous terms. By writing the line element as ${ }^{1}$

$$
d s^{2}=d t^{2}-\gamma_{i j}(\vec{x}, t) d x^{i} d x^{j},
$$

the Hamiltonian and momentum constraints take the form ${ }^{2}$

$$
\begin{gathered}
K^{2}-\operatorname{Tr} K^{2}+r=16 \pi G\left[(p+\rho) u_{0} u^{0}-p\right], \\
\nabla_{i} K-\nabla_{k} K_{i}^{k}=8 \pi G(p+\rho) u^{0} u_{i},
\end{gathered}
$$

\footnotetext{
*Electronic address: massimo.giovannini@cern.ch

${ }^{1}$ Note that, in this approach, $\gamma_{i j}(\vec{x}, t)$ contains 6 independent degrees of freedom corresponding to the correct number of initial conditions required for a general discussion of the problem.

${ }^{2}$ In the following, the overdot will denote a partial derivation with respect to the cosmic time coordinate.
}

where

$$
K_{i}^{j}=-\frac{1}{2} \gamma^{j k} \frac{\partial}{\partial t} \gamma_{k i}, \quad K=K_{i}^{i}, \quad \operatorname{Tr} K^{2}=K_{i}^{j} K_{j}^{i},
$$

and where $r=r_{i}^{i}$ is the trace of the spatial (instrinsic) curvature computed from the three-dimensional Ricci tensor in terms of $\gamma_{i j}$. The $(i j)$ components of Einstein equations are, instead,

$$
\frac{1}{\sqrt{\gamma}} \frac{\partial}{\partial t}\left(\sqrt{\gamma} K_{i}^{j}\right)-r_{i}^{j}=4 \pi G\left[-2(p+\rho) u_{i} u^{j}+(p-\rho) \delta_{i}^{j}\right]
$$

where $\gamma=\operatorname{det}\left(\gamma_{i j}\right)$.

Consider then the following expansion of the spatial metric, i.e.

$$
\gamma_{i j}(\vec{x}, t)=a^{2}(t)\left[\alpha_{i j}(\vec{x})+\beta_{i j}(\vec{x}, t)\right],
$$

when the term $\beta_{i j}(\vec{x}, t)$ contains the contribution of the gradients while $\alpha_{i j}(\vec{x})$ does not contain any gradient. Recalling that the inverse metric, to this order in the gradient expansion, is given by $\gamma^{i j}=\left[\alpha^{i j}-\beta^{i j}\right] / a^{2}(t)$, the extrinsic curvature (and its traces) can be readily computed. For instance, from Eqs. (4) and (6)

$$
K_{i}^{j}=-\left(H \delta_{i}^{j}+\frac{\dot{\beta}_{i}^{j}}{2}\right)
$$

where $H=\dot{a} / a$. Since from the momentum constraint (3) the velocity field is always of higher order in the gradient expansion, i.e.

$$
u^{0} u_{i}=\frac{\partial_{k} \dot{\beta}_{i}^{k}-\partial_{i} \dot{\beta}}{16 \pi G(p+\rho)},
$$

the contribution of the peculiar velocity field can be neglected in the remaining equations. This is not true necessarily to higher order in the gradient expansion.

Thus, recalling that $u_{0} u^{0}=1+\alpha^{i j} u_{i} u_{j} / a^{2}(t)$, and using Eqs. (4)-(7), Eqs. (2) and (5) can be written, respectively, as

$$
6 H^{2}+2 H \dot{\beta}+\frac{\mathcal{P}}{a^{2}}=16 \pi G \rho,
$$




$$
\begin{aligned}
& 2\left(\dot{H}+3 H^{2}\right) \delta_{i}^{j}+\ddot{\beta}_{i}^{j}+3 H \dot{\beta}_{i}^{j}+H \dot{\beta} \delta_{i}^{j}+\frac{2}{a^{2}} \mathcal{P}_{i}^{j} \\
& \quad=8 \pi G(\rho-p) \delta_{i}^{j},
\end{aligned}
$$

where we defined $r_{i}^{j}=\mathcal{P}_{i}^{j} / a^{2}$ (in this notation $\mathcal{P}_{i}^{j}$ and its traces are time independent).

Clearly, the trace-free part of Eq. (10) reduces to

$$
\begin{aligned}
& \left(\ddot{\beta}_{i}^{j}-\frac{1}{3} \ddot{\beta} \delta_{i}^{j}\right)+3 H\left(\dot{\beta}_{i}^{j}-\frac{1}{3} \dot{\beta} \delta_{i}^{j}\right) \\
& \quad=-\frac{2}{a^{2}}\left(\mathcal{P}_{i}^{j}-\frac{1}{3} \delta_{i}^{j} \mathcal{P}\right) .
\end{aligned}
$$

Equation (10) allows one to determine the gradient contribution to the energy density and the following relation,

$$
3\left(2 \dot{H}+3 H^{2}\right)+\ddot{\beta}+3 H \dot{\beta}+\frac{\mathcal{P}}{2 a^{2}}=-24 \pi G p,
$$

allows one to determine the gradient contribution to the pressure density.

Even if not strictly necessary, we can imagine to split $\rho$ and $p$ as

$$
\rho=\bar{\rho}+\tilde{\rho}, \quad p=\bar{p}+\tilde{p},
$$

where, from Eq. (10) $\bar{\rho}$ and $\bar{p}$ obey the usual Friedmann equations,

$$
\begin{gathered}
H^{2}=\frac{8 \pi G}{3} \bar{\rho}, \\
\left(2 \dot{H}+3 H^{2}\right)=-8 \pi G \bar{p}, \\
\dot{\rho}+3 H(\bar{\rho}+\bar{p})=0 .
\end{gathered}
$$

Equation (16) comes from the (0) component of the covariant conservation equation, i.e.

$$
\begin{gathered}
\frac{1}{\sqrt{\gamma}} \frac{\partial}{\partial t}\left\{\sqrt{\gamma}\left[(p+\rho) u_{0} u^{0}-p\right]\right\}-\frac{1}{\sqrt{\gamma}} \partial_{i}\left[\sqrt{\gamma}(p+\rho) u_{0} u^{i}\right] \\
-K_{k}^{\ell}\left[(p+\rho) u^{k} u_{\ell}+p \delta_{\ell}^{k}=0\right.
\end{gathered}
$$

Equation (17) also implies that $\tilde{\rho}$ and $\tilde{p}$ obey

$$
\frac{\partial \tilde{\rho}}{\partial t}+3 H(\tilde{\rho}+\tilde{p})+\frac{\dot{\beta}}{2}(\bar{\rho}+\bar{p})=0 .
$$

According to the same logic, Eq. (9) defines $\tilde{\rho}$ in terms of $\dot{\beta}$ and $\mathcal{P}$, i.e.

$$
2 H \dot{\beta}+\frac{\mathcal{P}}{a^{2}}=16 \pi G \tilde{\rho} .
$$

The fully inhomogeneous solution of the system can then be derived and it is

$$
\begin{gathered}
\beta_{i}^{j}(\vec{x}, t)=a^{-2-3 \epsilon} \mathcal{B}_{i}^{j}(\vec{x}) \\
K_{i}^{j}=-H\left[\delta_{i}^{j}-\frac{3 \epsilon+2}{2} a^{-2-3 \epsilon} \mathcal{B}_{i}^{j}(\vec{x})\right] .
\end{gathered}
$$

The space-dependent tensor $\mathcal{B}_{i}^{j}$ is related to the three- dimensional curvature tensor $\mathcal{P}_{i}^{j}$ by virtue of Eq. (10); the explicit result is

$$
\mathcal{P}_{i}^{j}=-\frac{H_{0}^{2}}{4}\left[(3 \epsilon+2)(3 \epsilon-2) \mathcal{B}_{i}^{j}-\left(3 \epsilon^{2}+12 \epsilon+4\right) \mathcal{B} \delta_{i}^{j}\right] .
$$

The physical significance of Eq. (22) is most easily understood by inverting Eq. (22), i.e.

$$
\mathcal{B}_{i}^{j}=\frac{4}{H_{0}^{2}\left(4-9 \epsilon^{2}\right)}\left[\mathcal{P}_{i}^{j}-\frac{3 \epsilon^{2}+12 \epsilon+4}{4(9 \epsilon+4)} \mathcal{P} \delta_{i}^{j}\right] .
$$

Equation (23) determines $\mathcal{B}_{i}^{j}$ as a function of the threedimensional spatial curvature computed from the $\alpha_{i j}(\vec{x})$. The form of $\alpha_{i j}$ is in a sense arbitrary. But once $\alpha_{i j}(\vec{x})$ is fixed, $\mathcal{B}_{i}^{j}(\vec{x})$ follow immediately from Eq. (23).

To derive Eqs. (22) and (23) the following parametrization for the scale factor and for the Hubble factor,

$$
a(t)=\left(\frac{t_{\mathrm{br}}-t}{t_{0}}\right)^{-[2 /(3 \epsilon)]}, \quad H(t)=H_{0} a^{(3 / 2) \epsilon},
$$

has been used. In Eq. (24) $t_{\text {br }}$ denotes the value of the cosmic time at the moment of the big-rip singularity. As a consequence of Eqs. (20)-(22), the energy density is

$$
\rho=\frac{3 H^{2}}{8 \pi G}\left[1+\frac{\epsilon}{2} a^{-2-3 \epsilon} \mathcal{B}(\vec{x})\right] .
$$

Since $\epsilon>0$, the relative contribution of the gradients to the energy density [second term inside the squared bracket of Eq. (25)] and to the extrinsic curvature [second term inside the squared bracket in Eq. (21)] vanishes asymptotically, for $t \rightarrow t_{\mathrm{br}}$. For instance, from Eq. (21), recalling Eq. (24)

$$
K_{i}^{j}=-H\left[\delta_{i}^{j}-\frac{3 \epsilon+2}{2}\left(\frac{t_{\mathrm{br}}-t}{t_{0}}\right)^{[2(2+3 \epsilon) /(3 \epsilon)]} \mathcal{B}_{i}^{j}(\vec{x})\right] .
$$

This property of big-rip singularities resembles what is known in the case of usual big-bang singularities where also the gradients decay though with a different power of the cosmic time coordinate. Big-bang singularities are argued to be homogeneous but also rather anisotropic [6]. As far as the big-rips are concerned, the situation may be very different.

To illustrate the last point, consider a given time $t_{*}$ at which gradients can be already neglected. This implies, in the notation of Eq. (6), that

$$
\alpha_{x x}=e^{2 A_{x}(t)}, \quad \alpha_{y y}=e^{2 A_{y}(t)}, \quad \alpha_{z z}=e^{2 A_{z}(t)},
$$

where, for simplicity, the tensor $\alpha_{i j}$ has been diagonalized. ${ }^{3}$

By solving Einstein equations in the absence of spatial curvature it is easy to show that

\footnotetext{
${ }^{3}$ Nondiagonal (or more general) forms of $\alpha_{i j}$ do not change the essence of the argument (see below).
} 


$$
A_{i}(t)=\frac{2 a\left(t_{*}\right) \mathcal{A}_{i}\left(t_{*}\right)}{3(\epsilon+2)}\left[1-\left(\frac{a}{a_{*}}\right)^{-(3 / 2)(\epsilon+2)}\right],
$$

where $\mathcal{A}_{i}$ are integration constants obeying $\sum_{i} \mathcal{A}_{i}=0$. Initial conditions have been fixed by requiring that $A_{i}\left(t_{*}\right)=0$. Clearly, recalling Eq. (24), in the limit $t \rightarrow$ $t_{\mathrm{br}}$, the second term in the squared bracket of Eq. (28) goes to zero and becomes then negligible as the big rip is approached. In the case of conventional big-bang singularities, the analog of the second term in the squared brackets evolves as $a^{3(w-1) / 2}$ with $0 \leq w<1$. In this case the contribution of the anisotropy clearly grows in the limit $a \rightarrow 0$.

As in the case of conventional big-bang singularities, the evolution of the anisotropy can also be studied in the more general case when spatial curvature is included [6]. Within our parametrization, the contribution of the curvature always decays as $a^{-2}$. Therefore, for $t \rightarrow t_{\mathrm{br}}$ the role of the curvature may be neglected. Again, this property is not realized in the vicinity of big-bang singularities. Actually, in the case of big-bang singularities the anisotropy grows as the bang is approached. For more general classes of Bianchi models, also the spatial curvature grows and this occurrence may induce the celebrated chaotic features and the related Belinsky-Khalatnikov-Lifshitz (BKL) oscillations [6].

Up to now we have considered the case where the big rip is caused by a perfect barotropic fluid with $w<-1$. Needless to say that, in the class of models previously investigated, the dominant energy condition is violated. In connection with this problem, both in brane-world models $[9,10]$ and in four-dimensional Friedmann-RobertsonWalker models [11-13] the possible occurrence of a different type of quiescent (or sudden) singularities has been emphasized. In sudden (future) singularities, the scale factor and its first derivative are both finite but higher derivatives of the scale factor may diverge. ${ }^{4}$ In some class of four-dimensional examples this behavior implies that, while the energy density is finite at the rip, the pressure density diverges. As correctly pointed out in [11], sudden singularities may occur without a violation of the dominant energy condition.

Consider then the example given in [11] of sudden singularities. The scale factor can be written as

$$
a(t)=\left(\frac{t}{t_{\mathrm{s}}}\right)^{q}\left(a_{\mathrm{s}}-1\right)+1-\left(1-\frac{t}{t_{\mathrm{s}}}\right)^{n} \text {. }
$$

For $1<n<2$ and $0<q \leq 1$, the solution is defined in the interval $0<t<t_{\mathrm{s}}$. For $t \rightarrow 0$ the model has a curvature (big-bang) singularity where the Hubble parameter and the energy density are both divergent. More interesting is the second singularity taking place for $t \rightarrow t_{\mathrm{s}}$. For $t \rightarrow t_{\mathrm{s}}$, the energy density and the Hubble parameter are both finite but the pressure density and the second time derivative of the scale factor are divergent.

The question we ought to address in the following concerns the nature of the gradient expansion in the vicinity of sudden singularities. From Eqs. (9) and (10) it can be argued that the contribution of the gradients to the quasiisotropic solution may be different if the scale factor behaves as in Eq. (29): in the limit $t \rightarrow t_{\mathrm{s}}$ both the scale factor and the Hubble rate are finite for the class of solutions given in Eq. (29). On the contrary, as previously discussed for big rips with $w<-1$, in the limit $t \rightarrow t_{\mathrm{br}}$, the scale factor and the Hubble rate are divergent [see Eq. (24)]. This difference is reflected in the contribution of the first-order gradients.

To avoid the proliferation of parameters, the attention will now be focused on a particular model belonging to the class defined by Eq. (29). Consider then the case $q=1 / 2$ and $n=3 / 2$. Indeed, the same qualitative results hold for all the models of the class described by Eq. (29) with the appropriate restrictions mentioned above. Let us also define, for notational convenience, the dimensionless variable $\tau=t / t_{\mathrm{s}}$. Then a particular solution of the fully inhomogeneous system including gradients can be easily obtained and expanded for $t \sim t_{\mathrm{s}}$, i.e. for $\tau \sim 1$. The most notable difference is that, in this case, pressure and energy density are completely independent and are not connected by any kind of equation of state. The final result for the extrinsic curvature can be written as

$$
K_{i}^{j}=-H(t) \delta_{i}^{j}-\lambda(t) \mathcal{B}_{i}^{j}(\vec{x})
$$

with $\mathcal{P}_{i}^{j}=t_{\mathrm{s}}^{-2} \mathcal{B}_{i}^{j}$ and where

$$
\begin{aligned}
& H(t)=\frac{1}{2 t_{\mathrm{s}}} \frac{b_{\mathrm{s}}+3 \sqrt{(1-\tau) \bar{\tau}}}{\left[1-(1-\tau)^{\frac{3}{2}}+b_{\mathrm{s}} \sqrt{\tau}\right] \sqrt{\tau}} \simeq \frac{1}{4 t_{\mathrm{s}} a_{\mathrm{s}}^{2}}\left[2 b_{\mathrm{s}} a_{\mathrm{s}}+6 a_{\mathrm{s}} \sqrt{1-\tau}+\left(a_{\mathrm{s}}+b_{\mathrm{s}}\right) b_{\mathrm{s}}(1-\tau)\right]+\mathcal{O}\left(|1-\tau|^{3 / 2}\right), \\
& \lambda(t)=-\frac{6 \sqrt{1-\tau}-3(-5+4 \sqrt{1-\tau}) \tau+10 b_{\mathrm{s}} \tau^{3 / 2}+6 \tau^{2} \sqrt{1-\tau}}{15 t_{\mathrm{s}}\left(1-\sqrt{1-\tau}+b_{\mathrm{s}} \sqrt{\tau}+\sqrt{1-\tau} \tau\right)^{3}},
\end{aligned}
$$

\footnotetext{
${ }^{4}$ Along a similar perspective but with a different chain of arguments, Ref. [14] also argues that the presence of a singularity in the future is not necessary even if the barotropic index is supernegative.
}

having defined, for notational convenience, $b_{\mathrm{s}}=a_{\mathrm{s}}-1$. As it can be appreciated from the last equations, the exact expressions are rather cumbersome, therefore, in the following, the result for the expansion in the limit $\tau \sim 1$ will be given directly. In particular, factorizing $H(t)$ in Eq. (30) 
and expanding the relative contribution of the gradients we have

$$
\begin{aligned}
K_{i}^{j}= & -H\left\{\delta_{i}^{j}+\left[-\frac{2}{3} \frac{2 a_{\mathrm{s}}+1}{b_{\mathrm{s}} a_{\mathrm{s}}^{2}}+\frac{2\left(2 a_{\mathrm{s}}+1\right)}{b_{\mathrm{s}}^{2} a_{\mathrm{s}}^{2}} \sqrt{1-\tau}\right.\right. \\
& +\frac{\left(a_{\mathrm{s}}+2\right)\left[1+a_{\mathrm{s}}^{2}\left(4 a_{\mathrm{s}}-13\right)-10 a_{\mathrm{s}}\right]}{3 b_{\mathrm{s}}^{3} a_{\mathrm{s}}^{3}}(1-\tau) \\
& \left.\left.+\mathcal{O}\left(|1-\tau|^{3 / 2}\right)\right] \mathcal{B}_{i}^{j}(\vec{x})\right\} .
\end{aligned}
$$

Clearly, in the limit $\tau \rightarrow 1$ (i.e. $t \rightarrow t_{\mathrm{s}}$ ) the contribution of the gradients, weighted by the space-dependent factor $\mathcal{B}(\vec{x})$, is not subleading. This behavior has to be contrasted with the case of big-rip singularities [see, for instance, Eqs. (21) and (26)] where for $t \rightarrow t_{\mathrm{br}}$ the gradient contribution is subleading.

In similar the form of $\rho$ and $p$ can also be derived and it is

$$
\begin{gathered}
\rho=\bar{\rho}\left\{1-\left[\frac{2\left(a_{\mathrm{s}}^{2}-2 a_{\mathrm{s}}-2\right)}{9 b_{\mathrm{s}}^{2} a_{\mathrm{s}}^{2}}+\frac{4\left(1+a_{\mathrm{s}}+a_{\mathrm{s}}^{2}\right)}{3 b_{\mathrm{s}}^{3} a_{\mathrm{s}}^{2}} \sqrt{1-\tau}\right.\right. \\
+\frac{2\left(-2+21 a_{\mathrm{s}}+17 a_{s}^{2}+47 a_{s}^{3}-3 a_{s}^{4}+a_{s}^{5}\right)}{9 a_{s}^{3} b_{s}^{4}}(1-\tau) \\
\left.\left.+\mathcal{O}\left(|1-\tau|^{3 / 2}\right)\right] \mathcal{B}(\vec{x})\right\}, \\
p=\bar{p}\left\{1+\left[\frac{\sqrt{1-\tau}}{3 a_{\mathrm{s}}}-\frac{a_{\mathrm{s}}^{2}-1}{18 a_{\mathrm{s}}^{2}}(1-\tau)\right.\right. \\
\left.\left.+\mathcal{O}\left(|1-\tau|^{3 / 2}\right)\right] \mathcal{B}(\vec{x})\right\}
\end{gathered}
$$

The exact expressions of $\bar{\rho}$ and $\bar{p}$ are a bit involved and then their expansion for $\tau \rightarrow 1$ will be given:

$$
\begin{aligned}
\bar{\rho} \simeq & \frac{3}{32 \pi G a_{\mathrm{s}}^{2} t_{\mathrm{s}}^{2}}\left[b_{\mathrm{s}}^{2}+6 b_{\mathrm{s}} \sqrt{1-\tau}\right. \\
& \left.+\frac{2 a_{\mathrm{s}}^{3}-5 a_{\mathrm{s}}^{2}+13 a_{\mathrm{s}}-1}{a_{\mathrm{s}}}(1-\tau)+\mathcal{O}\left(|1-\tau|^{3 / 2}\right)\right],
\end{aligned}
$$

$$
\begin{aligned}
\bar{p} \simeq & \frac{3}{16 \pi G a_{\mathrm{s}} t_{\mathrm{s}}^{2} \sqrt{1-\tau}}\left[1+\frac{a_{\mathrm{s}}^{2}-1}{2 a_{\mathrm{s}}^{2}} \sqrt{1-\tau}-\frac{3\left(a_{\mathrm{s}}-1\right)}{2 a_{\mathrm{s}}}\right. \\
& \left.\times(1-\tau)+\mathcal{O}\left(|1-\tau|^{3 / 2}\right)\right] .
\end{aligned}
$$

From Eq. (33) it can be deduced that as $t \rightarrow t_{\mathrm{s}}$ the gradients are not subleading. Moreover, from Eq. (35) one can also argue that for $t \rightarrow t_{\mathrm{s}}, \bar{\rho}$ is finite. On the contrary, in the same limit, i.e. $\tau \rightarrow 1$, the pressure density diverges [see Eq. (36)]. The amusing thing is that, in this case, the relative contribution of the gradients of Eq. (34) is subleading as $t \rightarrow t_{\mathrm{s}}$.

The analysis reported in a class of models parametrized by the scale factor of Eq. (29) suggests a crucial difference between sudden singularities and big rips. In the case of big rips the gradients are become progressively subleading as the singularity is approached. This statement holds for the extrinsic curvature, for the energy density, and for the pressure density. In the case of sudden singularities, the situation is different because the gradients do not decay in the extrinsic curvature and in the energy density (but they do decay in the pressure density). This occurrence suggests some general considerations on the possible chaotic behavior of sudden singularities and big rips.

To conclude, chaotic behavior in a given cosmological model is rather delicate. In the case of big-bang singularities (where the chaotic behavior is more plausible), the absence of spatial gradients seems to be a necessary condition to infer "homogeneous chaos" (like the one suggested by BKL oscillations [6]). This condition is partially violated in the case of sudden singularities, as explained in the previous paragraph. It would be tempting to conclude that in sudden singularities chaotic behavior, if present, is much weaker than in the case of big bangs and big rips. This speculation seems also to fit with the results of Ref. [15], where the geodesic properties of a class of sudden singularities have been analyzed.

In conclusion, let us summarize the main findings of the present investigation. In the first part it has been shown that if the dominant source of the background geometry is given by a perfect fluid with supernegative barotropic index (i.e. $w<-1$ ) then the contribution both of the spatial gradients and of the anisotropy tends to decay as the big-rip singularity is approached. For the more conventional big-bang singularities, the situation is a bit different: while gradients also decay in the vicinity of the big-bang, the anisotropy and the curvature may well grow and lead to some type of chaotic approach to the singularity.

We then moved to the analysis of sudden singularities. In the latter case the scale factor, the Hubble parameter, and the energy density are all finite as the singularity is approached but the pressure density diverges. In this situation we included the contribution of the gradients and showed in an explicit example (representative of a more general class of backgrounds) that the relative contribution of the gradients does not decay in the vicinity of the sudden singularity.

Various interesting generalizations are left for future works. The analysis of single fluid big-rip singularities can be generalized to a multifluid situation in analogy to what was recently discussed in the homogeneous and isotropic case [16]. In this situation it would be also interesting to discuss the fate of the anisotropy in more general Bianchi models. 
[1] A. A. Starobinsky, Gravitation Cosmol. 6, 157 (2000).

[2] R. R. Caldwell, Phys. Lett. B 545, 23 (2002).

[3] S. Nojiri and S. D. Odintsov, Phys. Rev. D 70, 103522 (2004).

[4] S. Nojiri and S. D. Odintsov, Phys. Lett. B 595, 1 (2004).

[5] M.P. Dabrowski, T. Stachowiak, and M. Szydlowski, Phys. Rev. D 68, 103519 (2003); M. P. Dabrowski, Phys. Rev. D 71, 103505 (2005).

[6] V. A. Belinskii and I. M. Khalatnikov, Sov. Phys. JETP 30, 1174 (1970).

[7] I. M. Khalatnikov, A. Yu. Kamenshchik, and A. A. Starobinsky, Classical Quantum Gravity 19, 3845 (2002).

[8] M. Giovannini, astro-ph/0506715.
[9] V. Sahni and Y. Shtanov, J. Cosmol. Astropart. Phys. 11 (2003) 014.

[10] V. Sahni and Y. Shtanov, Classical Quantum Gravity 19, L101 (2002).

[11] J. D. Barrow, Classical Quantum Gravity 21, L79 (2004).

[12] J. D. Barrow, Classical Quantum Gravity 21, 5619 (2004).

[13] J. D. Barrow and C. G. Tsagas, Classical Quantum Gravity 22, 1563 (2005).

[14] B. McInnes, J. High Energy Phys. 08 (2002) 029.

[15] L. Fernandez-Jambrina and R. Lazkoz, Phys. Rev. D 70, 121503 (2004).

[16] S. Nojiri, S. D. Odintsov, and S. Tsujikawa, Phys. Rev. D 71, 063004 (2005). 